\title{
Role of surface dimer dynamics in creating ordered organic-semiconductor interfaces
}

\author{
Robin L. Hayes \\ Department of Chemistry, New York University, New York, NY 10003 \\ Mark E. Tuckerman* \\ Department of Chemistry and Courant Institute of Mathematical Sciences \\ New York University, New York, NY 10003
}

\section{Supporting Information:}

Room temperature CMPD trajectories of 1,3-cyclohexadiene (CHD) reacting with the $\operatorname{Si}(100) 2 \times 1$ surface generated with VMD [1] In all cases: Si - yellow, C - blue/green, H - white, and Si surface dimers - red.

- S1: 4+2 intradimer adduct (A) shown in Figure 1, 3 and 4. (One unit cell, $1 \mathrm{~s}=225$ fs.)

- S2: $4+2$ interdimer, across row adduct (B) shown in Figure 1. (One unit cell, $1 \mathrm{~s}=$ 225 fs.)

- S3: $4+2$ interdimer, same row adduct with the $\mathrm{CH}_{2}$ groups over the trough $\left(\mathrm{C}_{t}\right)$ shown in Figure 1 and 3. (One unit cell, $1 \mathrm{~s}=225$ fs.)

- S4: $4+2$ interdimer, same row adduct with the $\mathrm{CH}_{2}$ groups over the row $\left(\mathrm{C}_{t}\right)$ shown in Figure 1 and 3. (One unit cell, $1 \mathrm{~s}=225$ fs.)

- S5: $2+2$ intradimer adduct (D) shown in Figure 1. (1.5 unit cell, $1 \mathrm{~s}=450$ fs.)

- S6: $2+2$ interdimer adduct (E) shown in Figure 1 and Figure 8. (4 unit cells, but only one blue unbound CHD, $1 \mathrm{~s}=450$ fs.)

- S7: Two CHD on the Si(100) surface with the long-lived intermediate shown in Figure 9. (1.5 unit cells, $1 \mathrm{~s}=450$ fs.)

- S8: Two CHD on the Si(100) surface, where the $2+2$ itradimer adduct (D) causes a Si dimer to flip, shown in Figure 10. (One unit cell, $1 \mathrm{~s}=225 \mathrm{fs}$.)

\section{References}

1. Humphrey, W.; Dalke, A.; Schulten, K. Journal of Molecular Graphics 1996, 14, $33-38$. 